(1)

netobertiver Journal of Nonlinear Mathematical Physics

\title{
On the Zeros of Polynomials Satisfying Certain Linear Second- Order ODEs Featuring Many Free Parameters
}

Francesco Calogero

To cite this article: Francesco Calogero (2013) On the Zeros of Polynomials Satisfying Certain Linear Second-Order ODEs Featuring Many Free Parameters, Journal of Nonlinear Mathematical Physics 20:2, 191-198, DOI:

https://doi.org/10.1080/14029251.2013.805565

To link to this article: https://doi.org/10.1080/14029251.2013.805565

Published online: 04 January 2021 


\title{
On the Zeros of Polynomials Satisfying Certain Linear Second-Order ODEs Featuring Many Free Parameters
}

\author{
Francesco Calogero \\ Dipartimento di Fisica, Universitá di Roma "La Sapienza" \\ Istituto Nazionale di Fisica Nucleare, Sezione di Roma \\ francesco.calogero@roma1.infn.it,francesco.calogero@uniromal.it \\ Received 19 January 2013 \\ Accepted 21 February 2013
}

\begin{abstract}
Certain techniques to obtain properties of the zeros of polynomials satisfying second-order ODEs are reviewed. The application of these techniques to the classical polynomials yields formulas which were already known; new are instead the formulas for the zeros of the (recently identified, and rather explicitly known) polynomials satisfying a (recently identified) second-order ODE which features many free parameters and only polynomial solutions. Some of these formulas have a Diophantine connotation. Techniques to manufacture infinite sequences of second-order ODEs featuring only polynomial solutions are also reported.
\end{abstract}

Keywords: zeros of polynomials, polynomials satisfying linear second-order ODEs, a linear second-order ODE with only polynomial solutions, Diophantine relations

\section{Introduction}

The properties of the zeros of polynomials satisfying linear second-order ODEs is a classical subject to which significant advances have been made over time by such mathematicians as J.C.F. Sturm, T.J. Stieltjes, G. Szegö and too many others to be listed here (yet let me heed the suggestion by an anonymous referee and refer the interested reader to a recent paper reporting new results on this topic but also having a review character indeed listing 48 references [1]). Many decades ago these findings were reviewed by Szegö in his classical treatise [2], and some decades ago many algebraic equations satisfied by the zeros of the classical polynomials were presented in [3], including sum rules and findings having a Diophantine connotation. Additional and related results of this kind are reviewed in Section 2.4 (entitled "Finite-dimensional representations of differential operators, Lagrangian interpolation, and all that") and Appendix D (entitled "Remarkable matrices and related identities") of [4], and in Appendix C (entitled "Diophantine findings and conjectures") of [5]. The main purpose of the present paper is to provide a few analogous results for the zeros of the (recently identified, and rather explicitly known) polynomials satisfying certain (recently identified) secondorder ODEs which feature many free parameters and only polynomial solutions [6]. To this end we report, in the following Section 2, firstly some general results for the zeros of polynomials satisfying linear second-order ODEs, and then the specific versions of these results for the zeros of the polynomials satisfying ODEs featuring only polynomial solutions, on which this paper is mainly focussed (indeed, techniques to manufacture infinite sequences of second-order ODEs having this 
property are also reported at the end of this section). The proofs of these findings are provided in the following Section 3. A terse Section 4 entitled Outlook completes this paper.

\section{Results}

In this paper we focus on (monic) polynomials of degree $N$,

$$
\psi_{N}(z)=\prod_{n=1}^{N}\left(z-z_{n}\right)=z^{N}+\sum_{m=1}^{N}\left(c_{m} z^{N-m}\right)=\sum_{m=0}^{N}\left(c_{m} z^{N-m}\right), \quad c_{0}=1,
$$

satisfying the linear second-order ODE

$$
a_{2}(z ; N) \psi_{N}^{\prime \prime}(z)+a_{1}(z ; N) \psi_{N}^{\prime}(z)+a_{0}(z ; N) \psi_{N}(z)=0 .
$$

Notation 2.1. Above and hereafter $N$ is a positive integer (large enough, see below), $\psi_{N}(z)$ is a (monic) polynomial of degree $N$ satisfying the ODE (2.2) (with appropriate assignments of the 3 functions $a_{2}(z ; N), a_{1}(z ; N)$ and $a_{0}(z ; N)$, depending parametrically on the integer $N$ and on other parameters, see below), $z_{n}$ are its $N$ zeros and $c_{m}$ its $N$ coefficients. Hereafter we assume for simplicity that the $N$ zeros $z_{n}$ are all different among themselves, $z_{n} \neq z_{m}$ if $n \neq m$; the results continue to hold if some zeros coincide, but generally only after taking appropriate limits. Indices such as $n, m, \ell$ take generally all integer values from 1 to $N$ (unless otherwise explicitly indicated). In (2.2) and hereafter primes appended to a function denote of course differentiations with respect to the argument of that function, $\psi_{N}^{\prime}(z) \equiv d \psi_{N}(z) / d z, \psi_{N}^{\prime \prime}(z) \equiv d^{2} \psi_{N}(z) / d z^{2}$.

Proposition 2.1. Provided the function $a_{2}(z ; N)$ does not vanish at the zeros $z_{n}$ and the function $a_{0}(z ; N)$ does not diverge at the zeros $z_{n}$,

$$
a_{2}\left(z_{n} ; N\right) \neq 0, \quad\left[a_{0}\left(z_{n} ; N\right)\right]^{-1} \neq 0,
$$

there hold the $N$ sum rules

$$
\sum_{\substack{\ell=1 \\ \ell \neq n}}^{N}\left(\frac{1}{z_{n}-z_{\ell}}\right)=-\frac{a_{1}\left(z_{n} ; N\right)}{2 a_{2}\left(z_{n} ; N\right)} .
$$

This result is rather elementary and presumably not new: for instance, in the case of the classical polynomials (Hermite, Laguerre, respectively Jacobi), see eqs. (3.3a), (4.2a) respectively (5.2a) of [3].

Corollary 2.1. There holds the sum rule

$$
\sum_{n=1}^{N}\left(\frac{a_{1}\left(z_{n}\right)}{a_{2}\left(z_{n}\right)}\right)=0
$$


Proposition 2.2. Let the $N \times N$ matrix $\underline{Q}$ be defined, componentwise, as follows:

$$
Q_{n n}=-\frac{z_{n} a_{1}\left(z_{n} ; N\right)}{2 a_{2}\left(z_{n} ; N\right)} ; \quad Q_{n m}=\frac{z_{n}}{z_{n}-z_{m}}, \quad n \neq m
$$

Then the $N$ eigenvalues of the $N \times N$ matrix $\underline{Q}$ are the $N$ integers from 0 to $N-1$,

$$
\underline{Q} \underline{v}^{(m)}=(m-1) \underline{v}^{(m)}
$$

and the corresponding eigenvectors $\underline{\underline{v}}^{(m)}$ are defined as follows:

$$
\underline{v}^{(m)} \equiv\left(v_{1}^{(m)}, \ldots, v_{n}^{(m)}\right), \quad v_{n}^{(m)}=\left(z_{n}\right)^{m} \prod_{\substack{\ell=1 \\ \ell \neq n}}^{N}\left(z_{n}-z_{\ell}\right)^{-1} .
$$

Notation 2.2. Hereafter $N \times N$ matrices such $\underline{Q}$ are denoted by underlined upper-case letters, and $N$-vectors by underlined lower case letters, so that for instance $\underline{v} \equiv\left(v_{1}, \ldots, v_{n}\right)$.

Next, we introduce the specific ODE on which we mainly focus in this paper, which is characterized by the following assignment of the functions $a_{2}(z ; N), a_{1}(z ; N)$ and $a_{0}(z ; N)$ in (2.2):

$$
a_{2}(z ; N)=1, a_{1}(z ; N)=-\left[\frac{N-1}{z}+R_{k}(z ; \underline{m}, \underline{x})\right], a_{0}(z)=\frac{N}{z} R_{k}(z ; \underline{m}, \underline{x}) .
$$

Notation 2.3. Hereafter, for any positive integer $K$, the rational function $R_{k}(z ; \underline{m}, \underline{x})$ of the independent variable $z$ is defined as follows,

$$
R_{k}(z ; \underline{m}, \underline{x})=\sum_{k=1}^{K}\left(\frac{m_{k}}{z-x_{k}}\right), \quad R_{k}^{\prime}(z ; \underline{m}, \underline{x})=-\sum_{k=1}^{K}\left[\frac{m_{k}}{\left(z-x_{k}\right)^{2}}\right],
$$

in terms of the $2 K$ parameters $m_{k}$ respectively $x_{k}$ which are the $K$ components of the two $K$-vectors $\underline{m}=\left(m_{1}, m_{2}, \ldots, m_{k}\right)$ respectively $\underline{x}=\left(x_{1}, x_{2}, \ldots, x_{k}\right)$. Hereafter we use the standard convention according to which a sum vanishes, and a product equals unity, if its upper limit is smaller than its lower limit. Note that the coefficients of the ODE (2.2) with (2.8) and (2.9a) become polynomial after multiplication of this ODE by the polynomial $z\left[\prod_{k=1}^{K}\left(z-x_{k}\right)\right]$ (of degree $1+K$ ).

In [6] it is shown that the general solution of the second-order ODE (2.2) with (2.8) and (2.9a) is a polynomial in $z$ of degree $N$, provided $K$ is (of course) a positive integer, the $K$ components $m_{k}$ of the $K$-vector $\underline{m}$ are all positive integers and their sum equals $N-1$,

$$
\underline{m} \equiv\left(m_{1}, \ldots, m_{k}\right) ; \quad m_{k} \text { integer, } m_{k}>0, \quad k=1, \ldots, K ; \quad \sum_{k=1}^{K} m_{k}=N-1,
$$

while the $K$ additional parameters provided by the $K$ components $x_{k}$ of the $K$-vector $\underline{x} \equiv\left(x_{1}, \ldots, x_{k}\right)$ are arbitrary numbers (possibly also complex; but obviously without loss of generality one may assume that these $K$ parameters $x_{k}$ are all different among themselves). 
Indeed in [6] it is shown that the general solution of the ODE (2.2) with (2.8) and (2.9) reads as follows:

$$
\begin{gathered}
\psi_{N}(z) \equiv \psi_{N}(z ; \underline{m}, \underline{x} ; \gamma)=z^{N} \varphi_{N}(z ; \underline{m}, \underline{x} ; \gamma), \\
\varphi_{N}(z ; \underline{m}, \underline{x} ; \gamma)=1+\frac{\gamma}{z} \sum_{\mu_{1}=0}^{m_{1}} \cdots \sum_{\mu_{k}=0}^{m_{k}}\left[\left(\prod_{k=1}^{K}\left[\left(\begin{array}{c}
m_{k} \\
\mu_{k}
\end{array}\right)\left(-\frac{x_{k}}{z}\right)^{\mu_{k}}\right]\right) \frac{1}{1+\sum_{\ell=1}^{K} \mu_{\ell}}\right],
\end{gathered}
$$

where $\gamma$ is an arbitrary (integration) constant. Here of course $\left(\begin{array}{c}m \\ \mu\end{array}\right)$ is the standard binomial coefficient,

$$
\left(\begin{array}{l}
m \\
\mu
\end{array}\right)=\frac{m !}{\mu !(m-\mu) !} .
$$

Remark 2.1. The general solution of the second-order ODE (2.2) should depend on two arbitrary parameters, one of which has however been fixed here, see (2.10), for consistency with the notationally convenient requirement (see (2.1)) that the polynomial $\psi_{N}(z)$ be monic.

Remark 2.2. The result reported above, see in particular (2.8) and (2.10), corresponds to the special case of the results of [6] with $J=0$. This restriction is justified by the fact that, in the more general case with $J>0$ (see [6]), the polynomial $\psi(z)$ introduced in [6] (of order $N+J$ ) features rather trivially $J$ zeros $y_{j}$ which can be arbitrarily assigned a priori; while the zeros $z_{n}$ of the polynomial (2.10) depend nontrivially on the $2 K$ parameters featured by the ODE (2.2) with (2.8) and (2.9), and also on the arbitrary (integration) constant $\gamma$. Moreover the sum rule of Proposition 2.1 obviously does not hold for the more general ODE considered in [6] (with $J>0$ ). Also note that the number $2 \mathrm{~K}$ of parameters can be arbitrarily large, but only if the degree $N$ is adequately large, since the last of the equations (2.9b) clearly entails that $N$ must be adequately larger than $K$.

Proposition 2.3. The $N \times N$ matrix $\underline{C}$ defined componentwise as follows,

$$
C_{n n}=\frac{N-1}{2}+\frac{z_{n}}{2} \sum_{k=1}^{K}\left(\frac{m_{k}}{z_{n}-x_{k}}\right), \quad C_{n m}=\frac{z_{n}}{z_{n}-z_{m}}, \quad n \neq m,
$$

features the $N$ integers from 0 to $N-1$ as its $N$ eigenvalues:

$$
\underline{C} \underline{v}^{(m)}=(m-1) \underline{v}^{(m)},
$$

with the corresponding eigenvectors $\underline{v}^{(m)}$ defined as above, see (2.7b). Here the parameters $m_{k}$ are $K$ (arbitrary) positive integers satisfying the single restriction

$$
\sum_{k=1}^{K} m_{k}=N-1
$$

the $K$ parameters $x_{k}$ are $K$ arbitrary numbers (possibly complex), and the $N$ numbers $z_{n}$ are the $N$ zeros of the polynomial of degree $N$ (2.10) (satisfying the ODE (2.2) with (2.8) and (2.9)).

Remark 2.3. Let us reemphasize that the $N$ zeros $z_{n}$ of the polynomial (2.10) depend (of course) on $N$ and on the $2 K$ parameters $m_{k}$ and $x_{k}$ which are featured by the ODE (2.2) with (2.8) and (2.9), and moreover on the additional arbitrary parameter $\gamma$ (which does not appear in this ODE but is 
featured by its solution (2.10)). And let us highlight the Diophantine connotation of Proposition 2.3, which we consider the main result of this paper.

Let us complete this section by displaying the ODE (2.2) with (2.8) and (2.9), as well as its solution (2.10), corresponding to the simplest assignments of the parameter $K$.

For $K=1$,

$$
\begin{aligned}
& z\left(z-x_{1}\right) \psi_{N}^{\prime \prime}-(N-1)\left(2 z-x_{1}\right) \psi_{N}^{\prime}+N(N-1) \psi_{N}=0 \\
& \begin{aligned}
\psi_{N}\left(N-1 ; x_{1} ; \gamma ; z\right) & =(1+\gamma) z^{N}-\gamma\left(z-x_{1}\right)^{N} \\
& =\left(\frac{x_{1}}{2}\right)^{N} p_{N}\left(0,0 ;-N \gamma ; \frac{2 z}{x_{1}}-1\right)
\end{aligned}
\end{aligned}
$$

with $\gamma$ and $x_{1}$ two arbitrary parameters. Here $p_{N}(0,0 ; \alpha ; y)$ is a para-Jacobi polynomial, see [7].

For $K=2$,

$$
\begin{array}{r}
z\left(z-x_{1}\right)\left(z-x_{2}\right) \psi_{N}^{\prime \prime}-\left\{2(N-1) z^{2}\right. \\
\left.-\left[\left(2 N-2-m_{1}\right) x_{1}+\left(N-1+m_{1}\right) x_{2}\right] z+(N-1) x_{1} x_{2}\right\} \psi_{N}^{\prime} \\
+N\left[(N-1) z-\left(N-1-m_{1}\right) x_{1}-m_{1} x_{2}\right] \psi_{N}=0, \\
+\gamma z^{N-1} \sum_{\mu_{1}=0}^{m_{1}} \sum_{\mu_{2}=0}^{N-1-m_{1}}\left[\left(\begin{array}{c}
m_{1} \\
\mu_{1}
\end{array}\right)\left(\begin{array}{c}
\left.N-1-m_{1}, N-1-m_{1} ; x_{1}, x_{2} ; \gamma ; z\right)=z^{N} \\
\mu_{2}
\end{array}\right) x_{1}^{\mu_{1}} x_{2}^{\mu_{2}} \frac{(-z)^{-\left(\mu_{1}+\mu_{2}\right)}}{1+\mu_{1}+\mu_{2}}\right],
\end{array}
$$

with $m_{1}$ an arbitrary integer in the range $0 \leqslant m_{1} \leqslant N-1$ and $\gamma, x_{1}, x_{2}$ three arbitrary parameters.

And finally let us proffer the following two remarks.

Remark 2.4. If a second-order ODE such as (2.2) admits a general solution which is (up to overall normalization) a monic polynomial $\psi_{N}(z)$ of arbitrary degree $N$, it is clearly easy to manufacture other analogous ODEs, say

$$
\tilde{a}_{2}(z ; N) \tilde{\psi}_{N}^{\prime \prime}(z)+\tilde{a}_{1}(z ; N) \tilde{\psi}_{N}^{\prime}(z)+\tilde{a}_{0}(z ; N) \tilde{\psi}_{N}(z)=0,
$$

having the same property to feature a general solution which is (up to normalization) a monic polynomial $\tilde{\psi}_{N}(z)$ of degree $N$. For instance such a (monic) polynomial solution could be

$$
\tilde{\psi}_{N}(z)=\frac{\psi_{N+1}^{\prime}(z)}{N+1}
$$

with the coefficients of the corresponding ODE (2.15)—obtained dividing (2.2) (with $N$ replaced by $N+1)$ by $a_{0}(z ; N+1)$, then differentiating, then multiplying by $a_{0}(z ; N+1)$ )-related to those 
of the ODE (2.2) as follows:

$$
\begin{gathered}
\tilde{a}_{2}(z ; N)=a_{2}(z ; N+1), \\
\tilde{a}_{1}(z ; N)=a_{1}(N+1 ; z)+a_{2}^{\prime}(N+1 ; z)-\frac{a_{0}^{\prime}(N+1 ; z) a_{2}(N+1 ; z)}{a_{0}(N+1 ; z)}, \\
\tilde{a}_{0}(z ; N)=a_{0}(N+1 ; z)+a_{1}^{\prime}(N+1 ; z)-\frac{a_{0}^{\prime}(N+1 ; z) a_{1}(N+1 ; z)}{a_{0}(N+1 ; z)} .
\end{gathered}
$$

The $N$ zeros $\tilde{z}_{n}$ of $\tilde{\psi}_{N}(z)$ coincide then with the $N$ extrema of $\psi_{N+1}(z)$.

Of course this procedure can be applied sequentially an arbitrary number of times, thereby producing an arbitrarily long sequence of ODEs whose general solution is a polynomial of degree $N$ in $z$; to each of these ODEs all the findings reported above therefore apply. Although in special cases—such as the sequence starting from (2.13)—all these equations might be identical, generally this shall not be the case: see for instance the sequence starting from the ODE (2.2) with (2.8) and (2.9) and $K \geqslant 2$, in particular (2.14).

It is also plain how to identify other second-order ODEs-more general than (2.15) with (2.16) - having the same property to only feature polynomial solutions of degree $N$ : for instance by replacing $\tilde{\psi}_{N}(z)$, see (2.16a), with $\check{\psi}_{N}(z)=\left[p_{M}(z) \psi_{N+1-M}(z)\right]^{\prime} /(N+1)$ with $p_{M}(z)$ an arbitrary (monic) polynomial of degree $M$ less than, say, $N-1$, and then following a procedure closely analogous to that indicated above after (2.16a).

Remark 2.5. The most general second-order ODE featuring only polynomial solutions $\psi(z)$ can be formulated as follows:

$$
\operatorname{det}\left(\begin{array}{ccc}
\psi(z) & p(z) & q(z) \\
\psi^{\prime}(z) & p^{\prime}(z) & q^{\prime}(z) \\
\psi^{\prime \prime}(z) & p^{\prime \prime}(z) & q^{\prime \prime}(z)
\end{array}\right)=0,
$$

with $p(z)$ and $q(z)$ two arbitrary (different) polynomials, i. e. (see (2.2)) $a_{2} \psi^{\prime \prime}+a_{1} \psi^{\prime}+a_{0} \psi=0$ with $a_{2}=w, a_{1}=-w^{\prime}, a_{0}=w^{\prime \prime}-p q^{\prime \prime \prime}+p^{\prime \prime \prime} q$ and $w=p q^{\prime}-p^{\prime} q$. Indeed clearly the general solution of this ODE reads

$$
\psi(z)=b p(z)+c q(z),
$$

with $b$ and $c$ two arbitrary (integration) constants. I was reminded of this fact by Robert Conte and Allan Fordy.

\section{Proofs}

In this Section 3 we prove the results reported in the preceding Section 2.

Firstly let us proof Proposition 2.1, see in particular (2.4). The very definition of the quantities $z_{n}$ as the $N$ zeros of the polynomial $\psi_{N}(z)$ - so that $\psi_{N}\left(z_{n}\right)=0$ - entails that the ODE (2.2) yields the $N$ relations

$$
a_{2}\left(z_{n}\right) \psi_{N}^{\prime \prime}\left(z_{n}\right)+a_{1}\left(z_{n}\right) \psi_{N}^{\prime}\left(z_{n}\right)=0 ;
$$

of course provided the function $a_{0}(z)$ does not diverge at $z=z_{n}$ (as assumed in the formulation of Proposition 2.1, see (2.3)). 
Notation 3.1. Of course above and hereafter notations such as $\psi_{N}^{\prime}\left(z_{n}\right)$ indicate the value of the function $\psi_{N}^{\prime}(z)$ at $z=z_{n}$.

Clearly the above relation can be rewritten as follows:

$$
\frac{\psi_{N}^{\prime \prime}\left(z_{n}\right)}{\psi_{N}^{\prime}\left(z_{n}\right)}=-\frac{a_{1}\left(z_{n}\right)}{a_{2}\left(z_{n}\right)}
$$

(of course, provided the function $a_{2}\left(z_{n}\right) \neq 0$, as assumed in the formulation of Proposition 2.1, see (2.3))

We then note that the first of the formulas (2.1) entails the identities

$$
\begin{gathered}
\psi_{N}^{\prime}(z)=\psi_{N}(z) \sum_{n=1}^{N}\left(z-z_{n}\right)^{-1} \\
\psi_{N}^{\prime \prime}(z)=\psi_{N}(z) \sum_{n=1}^{N}\left(z-z_{n}\right)^{-1} 2 \sum_{\substack{\ell=1 \\
\ell \neq n}}^{N}\left(z_{n}-z_{\ell}\right)^{-1} .
\end{gathered}
$$

The first of these two well-known identities - reported, with many analogous formulas, in Appendix A (entitled "Some useful identities") of [5]—is immediately obtained by logarithmic differentiation of the first of the formulas (2.1); and the second is as well easily obtained by differentiating the first and using some standard algebraic manipulations. Their ratio,

$$
\frac{\psi_{N}^{\prime \prime}(z)}{\psi_{N}^{\prime}(z)}=\frac{\sum_{m=1}^{N}\left(z-z_{m}\right)^{-1} 2 \sum_{\ell=1, \ell \neq m}^{N}\left(z_{m}-z_{\ell}\right)^{-1}}{\sum_{m=1}^{N}\left(z-z_{m}\right)^{-1}},
$$

clearly entails the simple relation

$$
\frac{\psi_{N}^{\prime \prime}\left(z_{n}\right)}{\psi_{N}^{\prime}\left(z_{n}\right)}=2 \sum_{\substack{\ell=1 \\ \ell \neq n}}^{N}\left(z_{n}-z_{\ell}\right)^{-1} .
$$

And this formula, together with (3.1b), implies (2.4). Proposition 2.1 is thereby proven.

Corollary 2.1 is immediately implied by summing (2.4) over $n$ from 1 to $N$ and noticing that the left-hand side then vanishes due to the antisymmetry of the summand under the exchange of the two indices $n$ and $\ell$.

Likewise the proof of Proposition 2.2 is an immediate consequence of (1), which implies that the $N \times N$ matrix $\underline{Q}$, see (2.6), coincides with the following $N \times N$ matrix $\underline{N}$ defined (componentwise) as follows:

$$
N_{n n}=z_{n} \sum_{\substack{\ell=1 \\ \ell \neq n}}^{N}\left(z_{n}-z_{\ell}\right)^{-1} ; \quad N_{n m}=\frac{z_{n}}{z_{n}-z_{m}}, \quad n \neq m .
$$

The fact that this matrix $\underline{N}$ - for any arbitrary assignment of the $N$ numbers $z_{n}$-has the $N$ integers $m-1$ from 0 to $N-1$ as its eigenvalues and the $N$ vectors $\underline{v}^{(m)}$ (see (2.7b)) as the corresponding eigenvalues, is (or should be) a well-known fact (see for instance eqs. (2.4.3-55b) and (2.4.3-56) of [4]).

And Proposition 2.3 is merely the special case of Proposition 2.2 corresponding to the ODE (2.2) with (2.8) and (2.9). 


\section{Outlook}

The approach utilized in this paper might be used to investigate analogous properties to those reported above, satisfied by the zeros of polynomial solutions of ODEs of higher order than two, and by the zeros of ODEs the solutions of which are not polynomial and feature an infinite number of zeros. These developments will be eventually reported if the results thereby obtained are deemed sufficiently novel and interesting to justify their publication.

\section{References}

[1] B. Shapiro, "Algebro-geometric aspects of Heine-Stieltjes theory", J. London Math. Soc. 83, 36-56 (2011).

[2] G. Szegö, Orthogonal polynomials, Amer. Math. Soc. Coll. Publ. 23, Providence, R. I., 1939 (see in particular section 6.7).

[3] S. Ahmed, M. Bruschi, F. Calogero, M. A. Olshanetsky and A. M. Perelomov, "Properties of the zeros of the classical polynomials and of Bessel functions", Nuovo Cimento 49B, 173-199 (1979).

[4] F. Calogero, Classical many-body problems amenable to exact treatments, Lecture Notes in Physics Monograph m66, Springer, Berlin, 2001.

[5] F. Calogero, Isochronous systems, Oxford University Press, Oxford, 2008; an updated paperback edition of this monograph has been published by OUP in September 2012.

[6] F. Calogero, "A linear second-order ODE with only polynomial solutions", J. Diff. Eqns. (submitted to, October 23, 2012).

[7] F. Calogero and Ge Yi, "Can the general solution of the second-order ODE characterizing Jacobi polynomials be polynomial?", J. Phys. A: Math. Theor. 45, 095206 (2012) (4 pages). doi: 10.1088/1751$8113 / 45 / 9 / 095206$. 